\title{
OBSERVABLES IN MODULAR FIELD THEORY
}

\author{
R. KLEEMAN*
}

(Received 23 October 1986; revised 8 January 1987)

\begin{abstract}
The observables of modular quantisation are studied from the point of view of locality. Such a study allows identification of possible Hamiltonians and also enables us to generalize the fundamental trilinear commutation relations of parafield theory. A comparison of modular field theory with a normal $U(m)$ gauge theory, begun in an earlier publication, is completed with the conclusion that the two are equivalent except that the former has certain restrictions on its observables.
\end{abstract}

\section{Introduction}

The best known form of generalised quantisation is parafield theory [4] and this has received much attention in the literature over the past twenty-five years [6]. Perhaps the most important result derived [2] has been that when a Fock representation is considered, parafield theory becomes essentially equivalent to a theory which is quantised normally but which has a $U(p)$ global gauge symmetry ( $p$ is the "order" of the parafield theory).

The possible observables of parafield theory have been studied from the point of view of locality by Ohnuki and Kamefuchi [13]. They have shown that locality restricts the possible algebraic form of the observables. Moreover when these restricted observables are expressed in terms of the normally quantised fields, it becomes clear that conditions of locality in parafield theory are the same as conditions of gauge invariance under certain orthogonal groups in the normally quantised theory.

* C.S.I.R.O. Division of Atmospheric Research, Private Bag 1, Mordialloc, Victoria 3195, Australia.

(๑) Copyright Australian Mathematical Society 1986, Serial-fee code 0334-2700/86 
In this paper it is proposed to study, also from the point of view of locality, the observables of a different scheme of quantisation known as modular field theory which was introduced by Green [5] about ten years ago. As in parafield theory, the locality constraints will be seen to impose algebraic constraints on modular observables. We shall demonstrate a set of observables obeying the locality contraints and use some of them to generalise the fundamental relation of parafield theory.

Modular field theory has been shown, like parafield theory, to have an ansatz solution whose algebraic structure is a colour algebra [15] (in the literature these are also referred to as generalised Lie algebras). This similarity of solution suggests a comparison of modular field theory with a normally quantised gauge theory may be useful in understanding the new quantisation. Some progress [9] has been made in this direction and in particular it has been shown that modular field theory of order $m$ possesses all the physically relevant "non-relativistic" states (those not involving anti-particles) for a $U(m)$ gauge theory. This analysis is continued below, mainly from the point of view of comparing observables in the two theories. In addition, in Section 5 we shall also consider the question of states involving anti-particles. We now present a review of the essentials of modular quantisation. Further details may be found in the author's previous publication [9].

\section{Basics of modular field theory}

We shall take the fundamental relations of modular field theory of order $m$ to be the following equal-time relations ${ }^{1}$ :

$$
\begin{aligned}
& \begin{array}{r}
\psi_{\alpha}\left(x_{1}\right) \psi_{\beta}^{*}\left(x_{2}\right) \psi_{\gamma}\left(x_{3}\right) \pm \psi_{\gamma}\left(x_{3}\right) \psi_{\beta}^{*}\left(x_{2}\right) \psi_{\alpha}\left(x_{1}\right) \\
=\delta\left(\mathbf{x}_{1}-\mathbf{x}_{2}\right) \delta_{\alpha \beta} \psi_{\gamma}\left(x_{3}\right) \pm \delta\left(\mathbf{x}_{2}-\mathbf{x}_{3}\right) \delta_{\beta \gamma} \psi_{\alpha}\left(x_{1}\right)
\end{array} \\
& \begin{array}{r}
\psi_{\alpha_{1}}^{*}\left(x_{1}\right) \psi_{\alpha_{2}}\left(x_{2}\right) \cdots \psi_{\alpha_{m+1}}\left(x_{m+1}\right) \pm \psi_{\alpha_{3}}\left(x_{3}\right) \cdots \psi_{\alpha_{m+1}}\left(x_{m+1}\right) \psi_{\alpha_{2}}\left(x_{2}\right) \psi_{\alpha_{1}}^{*}\left(x_{1}\right) \\
=\delta\left(\mathbf{x}_{1}-\mathbf{x}_{2}\right) \delta_{\alpha_{1} \alpha_{2}} \psi_{\alpha_{3}}\left(x_{3}\right) \cdots \psi_{\alpha_{m+1}}\left(x_{m+1}\right)
\end{array} \\
& \psi_{\alpha_{1}}\left(x_{1}\right) \cdots \psi_{\alpha_{m+1}}\left(x_{m+1}\right) \pm \psi_{\alpha_{m+1}}\left(x_{m+1}\right) \psi_{\alpha_{2}}\left(x_{2}\right) \cdots \psi_{\alpha_{m}}\left(x_{m}\right) \psi_{\alpha_{1}}\left(x_{1}\right)=0
\end{aligned}
$$

${ }^{1}$ The bold face spatial variables within delta functions implies that these variables are to be considered at equal times. The + signs refer to a Fermi like quantisation, while the - sigas refer to a Bose like quantisation. The Fermi case is considered below although a generalisation to the Bose case is straightforward. 
It should be observed that these relations are a generalisation of a set of relations discovered for parafield theory of order two [4] and in fact modular field theories of order one and two coincide (apart from a numerical factor) with parafield theories of the same order.

Another way of introducing modular field theory (and the way initially chosen by Green [5]) is to introduce a unitary operator $u$ satisfying $u^{m}=1$ and then define a superscript on the $\psi_{\alpha}(x)$ via

$$
\psi_{\alpha}^{(t)}(x)=u^{-t} \psi_{\alpha}(x) u^{t}
$$

The commutation relations are then assumed to take the form

$$
\begin{gathered}
\psi_{\alpha}^{*(r)}\left(x_{1}\right) \psi_{\beta}^{(s)}\left(x_{2}\right) \pm \psi_{\beta}^{(s+1)}\left(x_{2}\right) \psi_{\alpha}^{*(r+1)}\left(x_{1}\right)=\delta\left(\mathbf{x}_{1}-\mathbf{x}_{2}\right) \delta_{\alpha \beta} \delta^{r s}, \\
\psi_{\alpha}^{(r)}\left(x_{1}\right) \psi_{\beta}^{(s)}\left(x_{2}\right) \pm \psi_{\beta}^{(s-1)}\left(x_{2}\right) \psi_{\alpha}^{(r+1)}\left(x_{1}\right)=0 .
\end{gathered}
$$

It is quite straightforward to show that (2.3) implies (2.1) - by simple substitution and use of the relations (2.3). Whether the Fock representations of these two sets of relations coincide or not is, as yet, uncertain. Some light has been shed on this problem in the author's thesis [10] to which the reader is referred. The second set of relations, in fact, constitute an ansatz solution of the first set. To see this, consider the following non-singular linear transformation of the $\psi_{\alpha}^{(r)}(x)$ :

$$
\phi_{\alpha}^{(r)}(x) \equiv \frac{1}{\sqrt{m}} \sum_{s=0}^{m-1} \eta^{-r s} \psi_{\alpha}^{(s)}(x),
$$

where $\eta$ is the $m$ 'th primitive root of unity. When the inverse of this transformation is taken one is able to show that

$$
\psi_{\alpha}(x)=\frac{1}{\sqrt{m}} \sum_{r=0}^{m-1} \phi_{\alpha}^{(r)}(x) .
$$

In addition one can use (2.7) and (2.5) to derive the following relations for the $\phi_{\alpha}^{(r)}(x)$ :

$$
\begin{aligned}
\phi_{\alpha}^{(r)}\left(x_{1}\right) \phi_{\beta}^{(s)}\left(x_{2}\right) \pm \eta^{r-s} \phi_{\beta}^{(s)}\left(x_{2}\right) \phi_{\alpha}^{(r)}\left(x_{1}\right) & =0, \\
\phi_{\alpha}^{*(r)}\left(x_{1}\right) \phi_{\beta}^{(s)}\left(x_{2}\right) \pm \eta^{s-r} \phi_{\beta}^{(s)}\left(x_{2}\right) \phi_{\alpha}^{*(r)}\left(x_{1}\right) & =\delta_{\alpha \beta} \delta\left(\mathbf{x}_{1}-\mathbf{x}_{2}\right) \delta^{r s} \\
u^{-r} \phi_{\alpha}^{(s)}(x) u^{r} & \doteq \eta^{r s} \phi_{\alpha}^{(s)}(x)
\end{aligned}
$$

One can now show [9] that the above relations imply that the $\phi_{\alpha}^{(s)}(x)$ form a colour algebra. Equation (2.5) therefore gives an ansatz solution to modular field theory which is very similar to the ansatz solution of Green [4] for parafield theory. Since the transformation (2.4) is invertible, assumption of the relations (2.3) is equivalent to assuming the ansatz.

In the same way as parafield theory [2], we can introduce a Klein transformation [12] which transforms the $\phi_{\alpha}^{(s)}(x)$ into fields $\Phi_{\alpha}^{s}(x)$ which are quantised 
normally. Thus we have [9]

$$
\Phi_{\alpha}^{r}(x)=u^{r-1} \phi_{\alpha}^{(r)}(x)
$$

and it is straightforward to show that the $\Phi_{\alpha}^{r}(x)$ satisfy Bose or Fermi relations.

If we consider the Fock representation of the ansatz solution (2.5), then it is possible to compare the field theory of the $\Phi_{\alpha}^{r}(x)$ with the original modular field theory providing we assume that the vacuum state |\rangle and $u$ satisfy

$$
u|\rangle=|\rangle \text {. }
$$

The existence of a $u$ operator with the above properties has been demonstrated in [11].

\section{Locality constraints on observables}

In ordinary field theory the consideration of what constitutes an observable is far from resolved. As a consequence of this, we shall follow the approach used by Ohnuki and Kamefuchi [13] to consider the analogous problem in parafield theory. As was mentioned in the introduction this involves using locality conditions to restrict the possible algebraic form of observables.

The essential feature of this approach is that observables are defined in local regions of space. This is achieved as follows: Let $\mathrm{g}$ be a function of the fields $\psi\left(x_{1}\right), \psi\left(x_{2}\right), \ldots, \psi^{*}\left(y_{1}\right), \psi^{*}\left(y_{2}\right), \ldots$ and $h_{V}$ a function of $x_{1}, x_{2}, \ldots, y_{1}, y_{2}, \ldots$ which vanishes if any of its arguments lie spatially outside the region $V$. An observable $F(V)$ for the region $V$ is now defined to be of the form

$$
\begin{aligned}
& F(V)=\int_{\text {space }} h_{V} g\left(\psi\left(x_{1}\right), \psi\left(x_{2}\right), \ldots,\right. \\
&\left.\psi^{*}\left(y_{1}\right), \psi^{*}\left(y_{2}\right), \ldots\right) d x_{1} d x_{2} \cdots d y_{1} d y_{2} \cdots,
\end{aligned}
$$

with $x_{1}, x_{2}, \ldots, y_{1}, y_{2}, \ldots$ having the same time component.

A first requirement of our theory is that measurement of two observables defined at equal times in non-connected regions should be independent. This is simply an expression of the principle of causality and can be achieved through the following equal time equation

$$
\left[F(V), F^{\prime}\left(V^{\prime}\right)\right]_{-}=0 \quad V \sim V^{\prime},
$$

where $V \sim V^{\prime}$ means that $V$ and $V^{\prime}$ are disjoint. We shall refer to (3.2) as a condition of weak locality.

A stronger condition than (3.2) is the equal time relation

$$
[F(V), \hat{\psi}(x)]_{-}=0 \quad x \notin V,
$$


where $\hat{\psi}(x)=\psi(x)$ or $\psi^{*}(x)$. This relation ensures that measurement of $F(V)$ is unaffected by the existence of particles in regions which cannot have any causal influence ${ }^{2}$ on $V$. Condition (3.3) shall be referred to as strong locality. It is fairly clear that (3.3) implies (3.2), however, as we shall see below, the converse is certainly not true.

We turn now to the particular case of modular quantisation. We make the assumption here that the modular fields satisfy the conditions (2.2) and (2.5). In other words, we are considering the ansatz solution of the relations (2.1). We also restrict our attention here to the Fermi modular quantisation. These two assumptions will remain for the rest of this paper.

It is fairly easy to construct observables from modular fields which obey weak locality. An example is

$$
F(V)=\int_{\text {space }} h_{V}(x, y) \psi^{*}(y) \psi(x) d x d y .
$$

Relations (2.3) easily confirm that $\left[F(V), F\left(V^{\prime}\right)\right]_{-}=0$ for $V \sim V^{\prime}$. In general, however, these observables do not satisfy the condition of strong locality ${ }^{3}$. In order to consider the form of observables which are strongly local it proves convenient to allow them to be constructed from the ansatz fields $\phi^{*(r)}(x)$ and $\phi^{(r)}(x)$, or equivalently, by $(2.4)$, from the fields $\psi^{*(r)}(x)$ and $\psi^{(r)}(x)$. The following result now holds:

THEOREM 3.1. Observables $F(V)$ constructed from the ansatz fields obey strong locality if and only if

(i) They are functions of $\phi^{*(r)}(x) \phi^{(t)}(y), \phi^{\left(r_{1}\right)}\left(x_{1}\right) \cdots \phi^{\left(r_{q}\right)}\left(x_{q}\right)$ and its hermitean conjugate; where $q=m$ for $m$ even and $q=2 m$ for $m$ odd.

(ii) $u^{-1} F(V) u=F(V)$.

Proof. We first demonstrate the sufficiency of the two conditions: Using a Taylor series expansion of $g$ in (3.1), we may rewrite it, with the aid of (2.3) and a change of variables, as:

$$
\begin{aligned}
g\left(x_{1}, \ldots, y_{1}, \ldots\right)=\sum_{l, n, r_{i}, t_{j}} c_{l n}\left(r_{i}, x_{i}, t_{j}, y_{j}\right) \psi^{\left(r_{1}\right)}\left(x_{1}\right) \\
\\
\quad \ldots \psi^{\left(r_{1}\right)}\left(x_{l}\right) \psi^{*\left(t_{1}\right)}\left(y_{1}\right) \cdots \psi^{*\left(t_{n}\right)}\left(y_{n}\right) .
\end{aligned}
$$

\footnotetext{
${ }^{2}$ See Ohnuki and Kamefuchi [14], p. 88 for a more detailed discussion on this point.

${ }^{3}$ For $m=2$ candidates may be constructed [13]. For $m>2$ see Theorem 3.4 below.
} 
Now if we take $\psi(z)$ with $z \notin V$, we obtain after repeated use of (2.3)

$$
\begin{gathered}
\psi(z) F(V)=\int h_{V} \sum_{l, n, r_{i}, t_{j}} c_{l n} \psi^{\left(r_{1}-1\right)}\left(x_{1}\right) \cdots \psi^{\left(r_{l}-1\right)}\left(x_{l}\right) \psi^{*\left(t_{1}-1\right)}\left(y_{1}\right) \cdots \\
\psi^{*\left(t_{n}-1\right)}\left(y_{n}\right) \psi^{(l-n)}(z)(-1)^{n+l} d x_{1} \cdots d y_{1} \cdots
\end{gathered}
$$

By the use of condition (i) we have that $n-l \equiv 0 \bmod m$ and $n+l \equiv 0 \bmod 2$. It follows now from (2.2) and (3.6) that

$$
\psi(z) F(V)=u^{-1} F(V) u \psi(z)=F(V) \psi(z),
$$

when (ii) is used.

To demonstrate necessity we first rewrite (3.5) with the aid of (2.4):

$$
\begin{aligned}
g\left(x_{1}, \ldots, y_{1}, \ldots\right)= & \sum_{l, n, r_{i}, t_{j}} d_{l n}\left(r_{i}, x_{i}, t_{j}, y_{j}\right) \phi^{\left(r_{1}\right)}\left(x_{1}\right) \\
& \cdots \phi^{\left(r_{l}\right)}\left(x_{l}\right) \phi^{*\left(t_{1}\right)}\left(y_{1}\right) \cdots \phi^{*\left(t_{n}\right)}\left(y_{n}\right) .
\end{aligned}
$$

Secondly we regroup terms in this sum as follows:

$$
\begin{aligned}
F_{q t}^{(c)} \equiv & \sum_{\substack{x-y \equiv c \\
\bmod m}} \sum_{\substack{l-n \equiv q \\
\bmod m}} \sum_{\substack{t+n=t \\
\bmod 2}} d_{l n}\left(r_{i}, x_{i}, t_{j}, y_{j}\right) \phi^{\left(r_{1}\right)}\left(x_{1}\right) \cdots \phi^{\left(r_{l}\right)}\left(x_{l}\right) \\
& \cdot \phi^{*\left(t_{1}\right)}\left(y_{1}\right) \cdots \phi^{*\left(t_{n}\right)}\left(y_{n}\right)
\end{aligned}
$$

with $x \equiv \sum_{i=1}^{l} t_{i}$ and $y \equiv \sum_{i=1}^{n} r_{i}$. We now have the following commutation relations:

$$
\phi^{(b)}(z) h_{V} F_{q t}^{(c)}=\eta^{q b+c}(-1)^{t} h_{V} F_{q t}^{(c)} \phi^{(b)}(z)
$$

Since

$$
F(V)=\int h_{V} \sum_{c, q, t} F_{q t}^{(c)} d x_{1} \cdots d y_{1} \cdots,
$$

strong locality therefore demands that

$$
\int d \mathbf{x} d \mathbf{y} h_{V} \sum_{b, c, q, t}\left[1-\eta^{q b+c}(-1)^{t}\right] F_{q t}^{(c)} \phi^{(b)}(z)=0,
$$

where $d \mathbf{x}=d x_{1} d x_{2} \cdots$ and $d \mathbf{y}=d y_{1} d y_{2} \cdots$. Let $W$ be a region of space containing $z$ but not intersecting $V$. Define the following operator:

$$
u(W) \equiv \exp (A)
$$

with

$$
A=\frac{i 2 \pi}{m} \int \chi_{W}(x)\left[\sum_{r=0}^{m-1} r \phi^{*(r)}(x) \phi^{(r)}(x)\right] d x
$$


and

$$
\begin{aligned}
\chi_{W}(x) & =1 & & x \in W, \\
& =0 & & x \notin W .
\end{aligned}
$$

This operator will be unitary since $A$ is evidently anti-hermitean. Now

$$
\begin{aligned}
{\left[A, \phi^{(b)}(z)\right]_{-} } & =\frac{i 2 \pi}{m}\left[\int \chi_{W}(x)\left(\sum_{r=0}^{m-1} r \phi^{*(r)}(x) \phi^{(r)}(x)\right) d x, \phi^{(b)}(z)\right]_{-} \\
& =\frac{i 2 \pi}{m} \int \chi_{W}(x)-\delta(x-z) b \phi^{(b)}(x) d x \\
& =\frac{-i 2 \pi b}{m} \chi_{W}(z) \phi^{(b)}(z) .
\end{aligned}
$$

One of the Baker-Campbell-Hausdorff identities [3] allows us to then conclude that

$$
\begin{aligned}
u^{-1}(W) \phi^{(b)}(z) u(W) & =\exp \left(\frac{i 2 \pi b}{m} \chi_{W}(z)\right) \phi^{(b)}(z) \\
& =\eta^{b \chi_{W}(z)} \phi^{(b)}(z) .
\end{aligned}
$$

If (3.11) is premultiplied by $u^{-r}(W)$ and post-multiplied by $u^{r}(W)$ we obtain

$$
\int d \mathrm{x} d \mathrm{y} h_{V} \sum_{b, c, q, t} \eta^{r b}\left[1-\eta^{q b+c}(-1)^{t}\right] F_{q t}^{(c)} \phi^{(b)}(z)=0 .
$$

If we multiply this by $\eta^{-d r}$, sum over $r$ and use the following

$$
\sum_{r=0}^{m-1} \eta^{-d r} \eta^{r b}=\sum_{r=0}^{m-1} \eta^{r(b-d)}=m \delta_{b d},
$$

then we can conclude that

$$
\int d \mathbf{x} d \mathbf{y} h_{v} \sum_{c, q, t}\left[1-\eta^{q d+c}(-1)^{t}\right] F_{q t}^{(c)} \phi^{(d)}(z)=0 .
$$

We can use operators similar to $u(W)$ io eliminate the summations over $c, q$ and $t$ in the above equation. We now obtain

$$
\int d \mathbf{x} d \mathbf{y} h_{V}\left[1-\eta^{j d+i}(-1)^{s}\right] F_{j s}^{(i)} \phi^{(d)}(z)=0 .
$$

The above arguments are easily modifiable to the case $\psi^{*}(z)$ instead of $\psi(z)$. We are led then to

$$
\int d \mathbf{x} d \mathbf{y} h_{V}\left[1-\eta^{-j d-i}(-1)^{s}\right] F_{j s}^{(i)} \phi^{*(d)}(z)=0 .
$$

The bracketed quantity in (3.18) is the complex conjugate of the corresponding quantity in (3.19) and so therefore they vanish simultaneously. Suppose they vanish for all values of $d$. A little thought will show that this can only occur when 
$j=i=s=0$ (consider $d=1$ and $m-1$ and solve the relevant equations). Thus unless this situation occurs we may conclude that for some $d$

$$
\int d \mathbf{x} d \mathbf{y} h_{V} F_{j s}^{(i)} \phi^{(d)}(z)=\int d \mathbf{x} d \mathbf{y} h_{V} F_{j s}^{(i)} \phi^{*(d)}(z)=0 .
$$

Consider now the integral

$$
\int d \mathbf{x} d \mathbf{y} \int h_{V}\left(1-\chi_{V}(z)\right) F_{j s}^{(i)}\left(\phi^{*(d)}(z) \phi^{(d)}\left(z^{\prime}\right)+\phi^{(d)}\left(z^{\prime}\right) \phi^{*(d)}(z)\right) d z
$$

with $z^{\prime} \notin V$. By (3.20) this is zero, however by (2.6b) it is also

$$
\int d \mathbf{x} d \mathbf{y} \int h_{V}\left(1-\chi_{V}(z)\right) F_{j s}^{(i)} \delta\left(z-z^{\prime}\right) d z=\int d \mathbf{x} d \mathbf{y} h_{V} F_{j s}^{(i)}
$$

and so therefore we are led to

$$
\int d \mathbf{x} d \mathbf{y} h_{V} F_{j s}^{(i)}=0,
$$

whenever $i=j=s=0$ does not apply. Conditions (i) and (ii) now follow from (3.1), (3.7), (3.8) and (2.6c).

The question now arises as to the form of strongly local observables which are solely functions of the modular fields $\psi(x)$ and $\psi^{*}(x)$. This problem is partially solved in the following theorem:

THEOREM 3.2. Let us define the following polynomials of modular fields:

$$
\begin{aligned}
M\left(x_{1}, \ldots, x_{m-1}, y_{1}, \ldots, y_{m-1}\right) & \\
= & \sum_{\operatorname{perm}(1, \ldots, m-1)} \sum_{l=0}^{m-1} \psi\left(x_{m-1}\right) \cdots \psi\left(x_{m-1}\right) \psi^{*}\left(y_{m-1}\right) \\
& \times \cdots \psi^{*}\left(y_{1}\right) \psi\left(x_{1}\right) \cdots \psi\left(x_{m-l-1}\right)(-1)^{\prime} \\
B_{N}\left(x_{1}, \ldots, x_{N}\right)= & \sum_{\operatorname{cycl}(1, \ldots, N)}(-1)^{\operatorname{cycl}(N)} \psi\left(x_{1}\right) \cdots \psi\left(x_{N}\right) \\
C_{N}\left(x_{1}, \ldots, x_{m}\right)= & \sum_{\operatorname{cycl}(1, \ldots, N)} \psi\left(x_{1}\right) \cdots \psi\left(x_{N}\right) .
\end{aligned}
$$

An observable $F(V)$ is strongly local if the function $g$ in (3.1) is a function of the following modular field polynomials (and their hermitean conjugates): $M, C_{l m}\left(C_{l m}\right)^{\prime}$, $C_{l m}\left(C_{l m}^{*}\right)^{\prime}$ and when $k m$ is even, $B_{k m}$.

Proof. Contemplation of Theorem 3.1 shows that it is sufficient to show that the relevant field polynomials are invariant under $u$. The case of $B$ is considered firstly. 
It is easily seen from (2.2) and (2.3) that when $N$ is even and equal to $\mathrm{km}$ then

$$
\begin{aligned}
\psi\left(x_{N}\right) \psi\left(x_{1}\right) \cdots \psi\left(x_{N-1}\right) & =-\psi^{(-1)}\left(x_{1}\right) \cdots \psi^{(-1)}\left(x_{N-1}\right) \psi^{(N-1)}\left(x_{N}\right) \\
& =-\psi^{(-1)}\left(x_{1}\right) \cdots \psi^{(-1)}\left(x_{N-1}\right) \psi^{(-1)}\left(x_{N}\right)
\end{aligned}
$$

and therefore

$$
B_{N}\left(x_{1}, \ldots, x_{N}\right)=k \sum_{r=0}^{m-1} \psi^{(r)}\left(x_{1}\right) \cdots \psi^{(r)}\left(x_{N}\right)
$$

The form of the right-hand side of this equation now gives the desired result. The case of polynomials involving $C$ is proved in a similar way.

For the case of $M$ we move the fields $\psi\left(x_{1}\right), \ldots, \psi\left(x_{m-1-1}\right)$ in equation (3.22) to the left using equation (2.3) repeatedly. After a straightforward but tedious calculation we obtain the result:

$$
\begin{array}{r}
\sum_{\text {perm }} \sum_{q=0}^{m-1} \sum_{l=0}^{m-q-1} \sum_{n_{1}=0}^{k_{1}} \sum_{n_{2}=0}^{k_{2}} \cdots \sum_{n_{q}=0}^{k_{q}} X^{(0)}(m-l, m-1) X^{(-1)}\left(1, n_{1}\right) \\
X^{(-2)}\left(n_{1}+2, n_{1}+n_{2}+1\right) \cdots X^{(-q)}\left(t-n_{q}-1, t-2\right) \\
X^{(-q-1)}(t, m-l-1) Y^{(-l-q-1)}(m-1, t) Y^{(-l-q)}\left(t-2, t-n_{q}-1\right) \cdots \\
Y^{(-l-1)}\left(n_{1}, 1\right) \delta_{n_{1}+1}^{n_{1}+1} \delta_{n_{1}+n_{2}+2}^{n_{1}+n_{2}+2} \cdots \delta_{t-1}^{t-1}(-1)^{x}
\end{array}
$$

where

$$
\begin{gathered}
X^{(r)}(a, b) \equiv \psi^{(r)}\left(x_{a}\right) \cdots \psi^{(r)}\left(x_{b}\right) ; \quad Y^{(r)}(a, b) \equiv \psi^{*(r)}\left(y_{a}\right) \cdots \psi^{*(r)}\left(y_{b}\right) \\
t \equiv q+1+\sum_{i=1}^{q} n_{i} ; \quad x \equiv(l+1)(q-m+1)+l ; \\
\delta_{i}^{j} \equiv \delta\left(x_{j}-y_{i}\right) ; \quad k_{i} \equiv m-1-l-\sum_{j=1}^{i-1} n_{j} .
\end{gathered}
$$

This may now be rearranged by use of (2.3):

$$
\begin{aligned}
\sum & X^{(-t-1)}\left(1, n_{1}\right) \cdots X^{(-l-q)}\left(t-n_{q}-1, t-2\right) X^{(-t-q-1)}(t, m-1) \\
& Y^{(-t-q-1)}(m-1, t) \cdots Y^{(-t-1)}\left(n_{1}, 1\right) \delta_{n_{1}+1}^{n_{1}+1} \cdots \delta_{t-1}^{t-1}(-1)^{q-m-1} .
\end{aligned}
$$


By the rearrangement of the summations and by setting $n_{q+1} \equiv m-t$, we obtain

$$
\begin{array}{r}
\sum_{\text {perm }} \sum_{q=0}^{m-1}(-1)^{s} \sum_{\sum q_{i=1} n_{i}=s} \sum_{l=0}^{n_{q+1}} X^{(-l-1)}\left(1, n_{1}\right) \cdots X^{(-l-q-1)}\left(m-n_{q+1}, m-1\right) \\
Y^{(-l-q-1)}\left(m-1, m-n_{q+1}\right) \cdots Y^{(-l-1)}\left(n_{1}, 1\right) \\
\delta_{n_{1}+1}^{n_{1}+1} \cdots \delta_{m-n_{q+1}-1}^{m-n_{q+1}-1},
\end{array}
$$

where $s$ stands for $m-1-q$. Consider terms in this sum with fixed $n_{i}$ with $i=1, \ldots, q+1$. Corresponding to these terms are other terms with their $n_{i}$ being a cyclic permutation of these fixed values. After an appropriate permutation of the spatial indices these latter terms become

$$
\begin{array}{r}
\sum_{l=0}^{n_{j}} X^{(-l-1)}(f(j)+1, f(j+1)-1) \cdots X^{(-l-1+j-q)}\left(m-n_{q+1}, m-1\right) \\
X^{(-l-2+j-q)}\left(1, n_{1}\right) \cdots X^{(-l-q-1)}(f(j-1)+1, f(j)-1) \\
Y^{(-l-q-1)}(f(j)-1, f(j-1)+1) \cdots Y^{(-l-1)}(f(j+1)-1, f(j)+1) \\
\delta_{n_{1}+1}^{n_{1}+1} \cdots \delta_{m-n_{q+1}-1}^{m-n_{q+1}-1}
\end{array}
$$

where $f(j) \equiv j+\sum_{i=1}^{j} n_{i}$ with $j=1, \ldots, q$. This may be rearranged using (2.3) repeatedly:

$$
\begin{array}{r}
\sum_{l=0}^{n_{j}} X^{(-l-1+f(j))}\left(1, n_{1}\right) X^{(-l-2+f(j))}\left(n_{1}+2, n_{1}+n_{2}+1\right) \cdots \\
X^{(-l-1-q+f(j))}\left(m-n_{q+1}, m-1\right) Y^{(-l-1-q+f(j))}\left(m-1, m-n_{q+1}\right) \cdots \\
Y^{(-1-1+f(j))}\left(n_{1}, 1\right) \delta_{n_{1}+1}^{n_{1}+1} \cdots \delta_{m-n_{q+1}-1}^{m-n_{q+1}-1}
\end{array}
$$

Now as $l$ goes from 0 to $n_{j}$ the index $-l-1+f(j)$ goes from $-1+f(j)$ to $f(j-1)$ or when $j=1$, to 0 . In the original unpermuted term the corresponding index goes from -1 to $-1-n_{q+1}=t-1=q+\sum_{i-1}^{q} n_{i}=f(q)$. It is clear now that this index will cover all values $\bmod m$ when (3.29) is summed over all values of $j$ and added to the original term. Hence this sum will be invariant under $u$. A little thought about how the terms in (3.29) are produced will show that all terms in (3.27) may be grouped into such sums in a non-overlapping way. Hence $u^{-1} M u=M$. 
In the special cases of $m=2$ and $m=3$, the polynomial $M$ has the form

$$
\begin{gathered}
M\left(x_{1}, y_{1}\right)=\left[\psi\left(x_{1}\right), \psi^{*}\left(y_{1}\right)\right]_{-}, \\
M\left(x_{1}, x_{2}, y_{1}, y_{2}\right)=\sum_{\operatorname{perm}(1,2)} \psi^{*}\left(y_{2}\right) \psi^{*}\left(y_{1}\right) \psi\left(x_{1}\right) \psi\left(x_{2}\right) \\
\\
-\psi\left(x_{2}\right) \psi^{*}\left(y_{2}\right) \psi^{*}\left(y_{1}\right) \psi\left(x_{1}\right) \\
\\
+\psi\left(x_{1}\right) \psi\left(x_{2}\right) \psi^{*}\left(y_{2}\right) \psi^{*}\left(y_{1}\right) .
\end{gathered}
$$

In the case of $m=2$ we are dealing with parastatistics of order two and in that case the strong locality of $M$ follows directly from the fundamental commutation relation of paraquantisation [4]. This observation tends to suggest that a generalisation of this fundamental equation may be possible. This turns out to be the case as the following theorem shows:

THEOREM 3.3. The field polynomial $M$ given in equation (3.22) satisfies the following commutation relations:

$$
\begin{aligned}
{[M, \psi(z)]_{-}=} & \sum_{\text {perm }(1, \ldots, m-1)} \sum_{l=0}^{m-2}(-1)^{l+1} \delta\left(z-y_{m-l-1}\right) \psi^{*}\left(y_{m-l-2}\right) \cdots \psi^{*}\left(y_{1}\right) \\
& \quad \times \psi\left(x_{1}\right) \cdots \psi\left(x_{m-1}\right) \psi^{*}\left(y_{m-1}\right) \cdots \psi^{*}\left(y_{m-l}\right)
\end{aligned}
$$

Proof. We have firstly, the following interesting lemma:

\section{LEMMA.}

$$
\begin{aligned}
& {\left[\psi\left(x_{1}\right) \cdots \psi\left(x_{l}\right) \psi^{*}\left(y_{l}\right) \cdots \psi^{*}\left(y_{1}\right)\right.} \\
& \left.\psi^{*}\left(y_{m-1}\right) \cdots \psi^{*}\left(y_{l+1}\right) \psi\left(x_{l+1}\right) \cdots \psi\left(x_{m-1}\right)\right]_{-}=0 .
\end{aligned}
$$

Proof. We introduce the abbreviations $\psi\left(x_{i}\right)=x_{i}$ and $\psi^{*}\left(y_{i}\right)=y_{i}$. Now by (2.3) we have

$$
\begin{aligned}
& x_{1} \cdots x_{l} y_{l} \cdots y_{1} y_{m-1} \cdots y_{l+1} x_{l+1} \cdots x_{m-1} \\
& =x_{1} \cdots x_{l} y_{m-1}^{(l)} \cdots y_{l+1}^{(l)} y_{l}^{(l+1)} \cdots y_{1}^{(l+1)} x_{l+1} \cdots x_{m-1}(-1)^{l m} \\
& =y_{m-1} x_{1}^{(-1)} \cdots x l^{(-1)} y_{m-2}^{(l)} \cdots y_{l+1}^{(l)} y_{l}^{(l+1)} \cdots y_{1}^{(l+1)} x_{l+1} \cdots x_{m-1}(-1)^{l(m+l)} .
\end{aligned}
$$


Now for there to be any fields in the range $y_{m-2}^{(l)} \cdots y_{l+1}^{(l)}$, the index $l$ must be less than $m-2$. It follows from (2.3) that we may move $y_{m-2}^{(l)}$ to the left without picking up a delta function, thus:

$$
y_{m-1} y_{m-2} x_{1}^{(-2)} \cdots x_{l}^{(-2)} y_{m-3}^{(l)} \cdots y_{l+1}^{(l)} y_{l}^{(l+1)} \cdots y_{1}^{(l+1)} x_{l+1} \cdots x_{m-1}(-1)^{l m} .
$$

Obviously this argument can be extended until we obtain

$$
y_{m-1} \cdots y_{l+1} x_{1}^{(l+1)} \cdots x^{(l+1)} y_{l}^{(l+1)} \cdots y_{1}^{(l+1)} x_{l+1} \cdots x_{m-1} .
$$

By the use of an argument similar to the one just described we can move the fields $x_{l+1} \cdots x_{m-1}$ to the left obtaining

$$
y_{m-1} \cdots y_{l+1} x_{1}^{(l+1)} \cdots x^{(l+1)} x_{l+1}^{(l)} \cdots x_{m-1}^{(l)} y_{l} \cdots y_{1}(-1)^{l m}
$$

and finally this may be rewritten as

$$
y_{m-1} \cdots y_{l+1} x_{l+1} \cdots x_{m-1} x_{1} \cdots x_{l} y_{l} \cdots y_{1}
$$

which demonstrates the lemma.

As a corollary to the above lemma we have the following alternative form for $M$ :

$$
\begin{aligned}
M(x, y)= & \sum_{\operatorname{perm}(1, \ldots, m-1)} \sum_{l=0}^{m-1} \psi^{*}\left(y_{m-l-1}\right) \cdots \psi^{*}\left(y_{1}\right) \psi\left(x_{1}\right) \\
& \times \cdots \psi\left(x_{m-1}\right) \psi^{*}\left(y_{m-1}\right) \cdots \psi^{*}\left(y_{m-l}\right)(-1)^{l} .
\end{aligned}
$$

We begin the proof of Theorem 3.3 by demonstrating (3.33). By the use of the abbreviations introduced above we have, using (3.22),

$$
\begin{aligned}
& M(x, y) \psi^{*}(z)=\sum_{\text {perm }} \sum_{l=0}^{m-1}(-1)^{l} x_{m-l} \cdots x_{m-1} y_{m-1} \cdots y_{1} x_{1} \cdots x_{m-l-1} z \\
& =\sum(-1)^{m-1} x_{m-1} \cdots x_{m-1} y_{m-1} \cdots y_{1} z^{(l+1)} x_{1}^{(-1)} \cdots x_{m-l-1}^{(-1)} \\
& \quad+\sum_{\text {perm }} \sum_{l=0}^{m-2}(-1)^{l} x_{m-l} \cdots x_{m-1} y_{m-1} \cdots y_{1} x_{1} \cdots x_{m-l-2} \delta\left(z-x_{m-l-1}\right) .
\end{aligned}
$$

The first term in (3.37) is equal to

$$
\begin{aligned}
\sum x_{m-1} \cdots x_{m-1} z^{(l)} y_{m-1}^{(-1)} \cdots y_{1}^{(-1)} x_{1}^{(-1)} \cdots x_{m-l-1}^{(-1)} \\
=z \sum(-1)^{l} x_{m-l}^{(-1)} \cdots x_{m-1}^{(-1)} y_{m-1}^{(-1)} \cdots y_{1}^{(-1)} x_{1}^{(-1)} \cdots m_{m-l-1}^{(-1)} \\
=\psi^{*}(z) M^{(-1)}(x, y) \\
=\psi^{*}(z) M(x, y) .
\end{aligned}
$$

Consideration of the second term in (3.37) then gives equation (3.33). 
Now the hermitean conjugate of (3.22) is

$$
\begin{aligned}
M^{*}\left(x_{1}, \ldots, x_{m-1}, y_{1}, \ldots, y_{m-1}\right)= & \sum(-1)^{\prime} \psi^{*}\left(x_{m-1-1}\right) \cdots \psi^{*}\left(x_{1}\right) \psi\left(y_{1}\right) \ldots \\
& \times \psi\left(y_{m-1}\right) \psi^{*}\left(x_{m-1}\right) \cdots \psi^{*}\left(x_{m-1}\right) \\
= & M\left(y_{1}, \ldots, y_{m-1}, x_{1}, \ldots, x_{m-1}\right),
\end{aligned}
$$

where (3.36) has been used. We have now

$$
\begin{aligned}
{[M(x, y), \psi(z)]_{-} } & =-\left(\left[M^{*}(x, y), \psi^{*}(z)\right]_{-}\right)^{*} \\
& =-\left(\left[M(y, x), \psi^{*}(z)\right]_{-}\right)^{*}
\end{aligned}
$$

and then (3.32) follows from (3.33) and (3.39).

In the case $m=2$, the commutation relations given in (3.32) and (3.33) evidently have other solutions apart from simply $m=2$ modular field theory. These are, of course, the higher order parafield theories. One might expect, therefore, that the relations (3.32) and (3.33) will have further solutions when $m>2$. Whether this is so is, at present, unclear. In the special case of $m=3$ this author has attempted without success to find other ansatz solutions. This suggests that the above expectation may not be realized.

A further question deserving investigation is whether the new commutation relations can serve as the fundamental defining relations for modular field theory.

A classification of all strongly local observables remains an open question. In the case of $m=2$ the parafield classification [13] applies. In the more general setting the following theorem is of some interest:

ThEOREM 3.4. For $m>2$ there are no strongly local observables which are of second order or less in modular fields. For $m>4$ there are no such observables which are fourth order or less.

Proof. Consideration of Theorem 3.1 shows that first and third order polynomials are impossible for strongly local observables. We show now that second order polynomials are impossible for $m>2$.

By Theorem 3.1 such polynomials must involve both a $\psi$ and a $\psi^{*}$ and must therefore have the form

$$
F_{2}(V)=\int\left[a \psi\left(x_{1}\right) \psi^{*}\left(x_{2}\right)+b \psi^{*}\left(x_{2}\right) \psi\left(x_{1}\right)\right] d x_{1} d x_{2}+K
$$

where $K$ is a $c$-number and where $a$ and $b$ are functions of $x_{1}$ and $x_{2}$ which vanish when these variables are not in $V$. By the use of (2.5) and (2.6) this may be rewritten as

$$
F_{2}(V)=\int\left[\sum_{r, t}\left(a-\eta^{t-r} b\right) \phi^{(t)}\left(x_{1}\right) \phi^{*(r)}\left(x_{2}\right)\right] d x_{1} d x_{2}+K
$$


where $K$ is a $c$-number. When the notation of equation (3.8) in the proof of Theorem 3.1 is used, we may rewrite this as

$$
F_{2}(V)=\int\left[\sum_{v=0}^{m-1}\left(a-\eta^{-v} b\right) F_{00}^{(v)}\right] d x_{1} d x_{2}+K
$$

where we have

$$
F_{00}^{(v)}=\sum_{r-t=v} \phi^{(t)}\left(x_{1}\right) \phi^{*(r)}\left(x_{2}\right) .
$$

It follows from the proof of Theorem 3.1 that

$$
F_{v} \equiv \int\left(a-\eta^{-v} b\right) F_{00}^{(v)}=0 \quad v \neq 0 .
$$

Evaluate now

$$
0=F_{v} \phi^{(w)}\left(z_{2}\right)-\eta^{v} \phi^{(w)}\left(z_{2}\right) \cdot F_{v} \equiv G_{v-w}
$$

and then

$$
G_{v-w} \phi^{*(w-v)}\left(z_{1}\right)+\eta^{v-w} \phi^{*(w-v)}\left(z_{1}\right) G_{v-w} .
$$

After these calculations are carried out, we obtain the equation

$$
a\left(z_{1}, z_{2}\right)-\eta^{-v} b\left(z_{1}, z_{2}\right)=0 \quad v \neq 0 .
$$

If $v$ can take on more than one value, as it can when $m>2$, then this implies that $a=b=0$ which shows that $F_{2}(V)=0$.

If $m>4$ it is clear from Theorem 3.1 that the only possible strongly local observables of fourth order involve two $\psi$ and two $\psi^{*}$ fields. Some thought as to the possible permutations of these fields leads us to conclude that such observables must have the form ${ }^{4}$

$$
\begin{aligned}
& F_{4}(V)=\int d x_{1} d x_{2} d y_{1} d y_{2}\left[a_{1} \psi\right.\left(x_{1}\right) \psi\left(x_{2}\right) \psi^{*}\left(y_{1}\right) \psi^{*}\left(y_{2}\right) \\
&+a_{2} \psi\left(x_{1}\right) \psi^{*}\left(y_{1}\right) \psi\left(x_{2}\right) \psi^{*}\left(y_{2}\right) \\
&+a_{3} \psi^{*}\left(y_{1}\right) \psi\left(x_{1}\right) \psi\left(x_{2}\right) \psi^{*}\left(y_{2}\right) \\
&+a_{4} \psi^{*}\left(y_{1}\right) \psi\left(x_{1}\right) \psi^{*}\left(y_{2}\right) \psi\left(x_{2}\right) \\
&\left.+a_{5} \psi^{*}\left(y_{1}\right) \psi^{*}\left(y_{2}\right) \psi\left(x_{1}\right) \psi\left(x_{2}\right)\right] \\
&+\int d x d y\left[b_{1} \psi(x) \psi^{*}(y)+b_{2} \psi^{*}(y) \psi(x)\right],
\end{aligned}
$$

where $a_{i}$ and $b_{i}$ are functions vanishing when their arguments lie outside $V$.

\footnotetext{
${ }^{4}$ We are using $\psi^{*}\left(y_{1}\right) \psi\left(x_{1}\right) \psi\left(x_{2}\right) \psi^{*}\left(y_{2}\right)=\psi\left(x_{2}\right) \psi^{*}\left(y_{2}\right) \psi^{*}\left(y_{1}\right) \psi\left(x_{1}\right)$ which is a trivial consequence of equations (2.3).
} 
When (2.2), (2.3) and (2.5) are used, we can rewrite the terms in the first bracket (apart from a $c$-number) as

$$
\begin{array}{r}
\sum_{r_{1}, r_{2}, t_{1}, t_{2}} \Lambda\left(r_{1}, r_{2}, t_{1}, t_{2}\right) \phi^{\left(t_{1}\right)}\left(x_{1}\right) \phi^{\left(t_{2}\right)}\left(x_{2}\right) \phi^{*\left(r_{1}\right)}\left(y_{1}\right) \phi^{*\left(r_{2}\right)}\left(y_{2}\right) \\
+\sum_{r, t} \sum_{i, j=1}^{2} T^{i j}(r, t) \phi^{(t)}\left(x_{i}\right) \phi^{*(r)}\left(y_{j}\right)
\end{array}
$$

where

$$
\begin{aligned}
\Lambda\left(r_{1}, r_{2}, t_{1}, t_{2}\right) & =a_{1}-\eta^{t_{2}-r_{1}} a_{2}+\eta^{v+r_{1}-r_{2}} a_{3}-\eta^{v+t_{2}-r_{1}} a_{4}+\eta^{2 v} a_{5}, \\
v & =t_{1}+t_{2}-r_{1}-r_{2}
\end{aligned}
$$

and $T^{i j}$ are products of delta functions and the $a_{k}$. By the use of a change of variables, the second integral in (3.45) can be combined with the second term from (3.46) and we may write (3.45) as

$$
\begin{aligned}
F_{4}(V)= & \int d x_{1} d x_{2} d y_{1} d y_{2} . \\
& \times\left[\sum_{r_{i}, t_{i}} \Lambda\left(r_{1}, r_{2}, t_{1}, t_{2}\right) \phi^{\left(t_{1}\right)}\left(x_{1}\right) \phi^{\left(t_{2}\right)}\left(x_{2}\right) \phi^{*\left(r_{1}\right)}\left(y_{1}\right) \phi^{*\left(r_{2}\right)}\left(y_{2}\right)\right. \\
& \left.+\sum_{r, t} \sum_{i, j} U^{i j}(r, t) \phi^{(t)}\left(x_{i}\right) \phi^{*(r)}\left(y_{j}\right)\right]+K
\end{aligned}
$$

where $U^{i j}$ are products of delta functions the $a_{k}$ and the $b_{k}$.

By the use of a similar proof to the one used for second order observables above, we may conclude, using the proof of Theorem 3.1, that

$$
\int d x_{1} d x_{2} d y_{1} d y_{2} F_{00}^{(v)}=0 \quad v \neq 0
$$

with

$$
\begin{aligned}
F_{00}^{(v)}= & \sum_{t_{1}+t_{2}-r_{1}-r_{2}=v} \Lambda \phi^{\left(t_{1}\right)}\left(x_{1}\right) \phi^{\left(t_{2}\right)}\left(x_{2}\right) \phi^{*\left(r_{1}\right)}\left(y_{1}\right) \phi^{*\left(r_{2}\right)}\left(y_{2}\right) \\
& +\sum_{t-r=v} \sum_{i j} U^{i j} \phi^{(t)}\left(x_{i}\right) \phi^{*(r)}\left(y_{j}\right) .
\end{aligned}
$$

We introduce the following notation:

$$
F_{v} \equiv \int F_{00}^{(v)} d x_{1} d x_{2} d y_{1} d y_{2}, \quad \Lambda_{t_{1} t_{2} r_{1} r_{2}}^{x_{1} x_{2} y_{1} y_{2}} \equiv \Lambda\left(r_{1}, r_{2}, t_{1}, t_{2}\right)
$$

where $\Lambda$ is evaluated at $x_{1}, x_{2}, y_{1}, y_{2}$. 
Now when (3.49), (3.50) and (2.6) are used we obtain

$$
\begin{aligned}
& 0=\phi^{(a)}\left(z_{1}\right) F_{v}-\eta^{-v} F_{v} \phi^{(a)}\left(z_{1}\right) \\
& =-\eta^{-v}\left[\int \sum_{t_{1}+t_{2}-r=v+a}\left[\Lambda_{t_{1} t_{2} r a}^{x_{1} x_{2} y z_{1}}-\eta^{a-r} \Lambda_{t_{1} t_{2} a r}^{x_{1} x_{2} z_{1} y}\right]\right. \\
& \cdot \phi^{\left(t_{1}\right)}\left(x_{1}\right) \phi^{\left(t_{2}\right)}\left(x_{2}\right) \phi^{*(r)}(y) d x_{1} d x_{2} d y \\
& \left.\quad+\int U^{i j} \phi^{(v+a)}\left(x_{i}\right) \delta\left(z_{1}-y_{j}\right) d x_{1} d x_{2} d y_{1} d y_{2}\right] \\
& \equiv-\eta^{-v} G_{v+a} \quad v \neq 0 .
\end{aligned}
$$

Similarly we have$$
\begin{aligned}
0 & =\phi^{(b)}\left(z_{2}\right) G_{v+a}+\eta^{b-v-a} G_{v+a} \phi^{(b)}\left(z_{2}\right) \\
& =-\eta^{-v-a} \int \sum_{t_{1}+t_{2}=v+a+b}\left[\Lambda_{t_{1} t_{2} b a}^{x_{1} x_{2} z_{2} z_{1}}-\eta^{a-b} \Lambda_{t_{1} t_{2} a b}^{x_{1} x_{2} z_{2}}\right] \\
& =-\phi^{\left(t_{1}\right)}\left(x_{1}\right) \phi^{\left(t_{2}\right)}\left(x_{2}\right) d x_{1} d x_{2} \\
& \equiv-\eta^{-v-a} H_{v+a+b} \quad v \neq 0 .
\end{aligned}
$$

If we continue in this manner we obtain

$$
\begin{aligned}
0 & =\phi^{*(c)}\left(z_{3}\right) H_{v+a+b}-\eta^{v+a+b-2 c} H_{v+a+b} \phi^{*(c)}\left(z_{3}\right) \\
= & -\eta^{v+a+b-2 c} \int d x\left[\Lambda_{d c b a}^{x_{3} z_{2} z_{1}}-\eta^{a-b} \Lambda_{d c a b}^{x_{3} z_{2} z_{2}}-\eta^{c-d} \Lambda_{c d b a}^{z_{3} x z_{2} z_{1}}\right. \\
& \left.\quad+\eta^{c+a-b-d} \Lambda_{c d a b}^{z_{3} x z_{2} z_{2}}\right] \phi^{(d)}(x) \\
& =-\eta^{d} J_{d} \quad d+c-a-b \neq 0,
\end{aligned}
$$

where we have defined $d \equiv v+a+b-c$. Finally taking the anticommutator of $J_{d}$ with $\phi^{*(d)}\left(z_{4}\right)$ we obtain

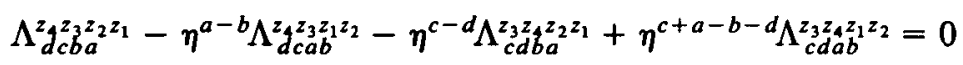

for $d+c-a-b \neq 0$. By the use of (3.47) and the notation

$$
a_{i}^{4321} \equiv a_{i}\left(z_{4}, z_{3}, z_{2}, z_{1}\right) \text {, }
$$

we obtain the equation

$$
\begin{aligned}
& a_{1}^{4321}-\eta^{a-b} a_{1}^{4312}-\eta^{c-d} a_{1}^{3421}+\eta^{c+a-b-d} a_{1}^{3412} \\
& -\eta^{c-b}\left(a_{2}^{4321}-a_{2}^{4312}-a_{2}^{3421}+a_{2}^{3412}\right) \\
& +\eta^{d+c-a-b}\left(\eta^{a-b} a_{3}^{4321}-a_{3}^{4312}-\eta^{c+a-b-d} a_{3}^{3421}+\eta^{c-d} a_{3}^{3412}\right) \\
& -\eta^{d+c-a-b} \eta^{c-b}\left(a_{4}^{4321}-a_{4}^{4312}-a_{4}^{3421}+a_{4}^{3412}\right) \\
& +\eta^{2(d+c-a-b)}\left(a_{5}^{4321}-\eta^{a-b} a_{5}^{4312}-\eta^{c-d} a_{5}^{3421}+\eta^{c+a-b-d} a_{5}^{3412}\right)=0
\end{aligned}
$$

when $d+c-a-b \neq 0$. 
If we take $d=a+b-c+1$ and $d=a+b-c+2$ and remember that $m>2$ then we may subtract the resulting equations obtaining

$$
\begin{gathered}
\left(\eta^{-1}-\eta^{-2}\right)\left[\eta^{2(c-b)} a_{1}^{3412}-\eta^{2 c-a-b} a_{1}^{3421}\right]+\left(\eta-\eta^{2}\right)\left[\eta^{a-b} a_{3}^{4321}-a_{3}^{4312}\right] \\
-\left(\eta-\eta^{2}\right) \eta^{c-b}\left[a_{4}^{4321}-a_{4}^{4312}-a_{4}^{3421}+a_{4}^{3412}\right] \\
+\left(\eta^{2}-\eta^{4}\right)\left[a_{5}^{4321}-\eta^{a-b} a_{5}^{4312}\right] \\
-\left(\eta-\eta^{2}\right)\left[\eta^{2 c-a-b} a_{5}^{3421}-\eta^{2(c-b)} a_{5}^{3412}\right]=0 .
\end{gathered}
$$

If we let $a=0,1$ and subtract the resulting equations we obtain

$$
\begin{aligned}
& \left(\eta^{-1}-\eta^{-2}\right)\left(\eta^{-1}-1\right) \eta^{2 c-b} a_{1}^{3421}+\left(\eta-\eta^{2}\right)(1-\eta) \eta^{-b} a_{3}^{4321} \\
& +\left(\eta^{2}-\eta^{4}\right)(\eta-1) \eta^{-b} a_{5}^{4312}+\left(\eta-\eta^{2}\right)\left(\eta^{-1}-1\right) \eta^{2 c-b} a_{5}^{3421}=0 .
\end{aligned}
$$

If $c=0,1$ is then substituted, subtraction of these equations gives

$$
a_{1}^{3421}\left(\eta^{-1}-\eta^{-2}\right)+a_{5}^{3421}\left(\eta-\eta^{2}\right)=0 .
$$

The above procedure may be repeated, in the case of $m>3$, with $d=a+b-c$ -1 and $d=a+b-c+1$ in (3.56). The resulting expression is

$$
a_{1}^{3421}\left(\eta^{-1}-\eta\right)+a_{5}^{3421}\left(\eta-\eta^{-1}\right)=0
$$

or in otherwords $a_{1}^{3421}=a_{5}^{3421}$. Substitution of this into (3.59) gives

$$
a_{1}^{3421}\left(\eta^{-1}-\eta^{-2}+\eta+\eta^{2}\right)=0
$$

which for $m>3$ implies that $a_{1}^{3421}=0=a_{5}^{3421}$. Substitution into (3.58) gives also $a_{3}^{4321}=0$. Equations (3.57) and (3.56) finally give

$$
\begin{aligned}
& a_{4}^{4321}-a_{4}^{4312}-a_{4}^{3421}+a_{4}^{3412}=0, \\
& a_{2}^{4321}-a_{2}^{4312}-a_{2}^{3421}+a_{2}^{3412}=0 .
\end{aligned}
$$

By the use of the first equation of (2.1) and its hermitean conjugate the following identity may be derived:

$$
\begin{aligned}
\psi\left(x_{1}\right) \psi^{*}\left(y_{1}\right) \psi\left(x_{2}\right) \psi^{*}\left(y_{2}\right)-\psi\left(x_{2}\right) \psi^{*}\left(y_{1}\right) \psi\left(x_{1}\right) \psi^{*}\left(y_{2}\right) & -\psi\left(x_{1}\right) \psi^{*}\left(y_{2}\right) \psi\left(x_{2}\right) \psi^{*}\left(y_{1}\right)+\psi\left(x_{2}\right) \psi^{*}\left(y_{2}\right) \psi\left(x_{1}\right) \psi^{*}\left(y_{1}\right) \\
\equiv & 4 \psi\left(x_{1}\right) \psi^{*}\left(y_{1}\right) \psi\left(x_{2}\right) \psi^{*}\left(y_{2}\right)-3 \delta\left(x_{2}-y_{1}\right) \psi\left(x_{1}\right) \psi^{*}\left(y_{2}\right) \\
& +\delta\left(x_{1}-y_{2}\right) \psi\left(x_{2}\right) \psi^{*}\left(y_{1}\right)-\delta\left(x_{1}-y_{1}\right) \psi\left(x_{2}\right) \psi^{*}\left(y_{2}\right) \\
& -\delta\left(x_{2}-y_{2}\right) \psi\left(x_{1}\right) \psi^{*}\left(y_{1}\right) .
\end{aligned}
$$

If we multiply the left hand side by $a_{2}\left(x_{1}, x_{2}, y_{1}, y_{2}\right)$ and integrate over the four variables we may, after a change of variables in the final three integrals and use of (3.60), conclude that the result is zero. Examination of the right hand side of the identity will then show that the second term in (3.54) becomes a quadratic term. 
A similar argument holds for the fourth term and so we conclude that $F_{4}(V)$ must be a second order polynomial and hence, from the first part of the proof, zero.

It should be remarked at this point that the above four theorems are easily modified to deal with derivative fields.

\section{Relationship to a normal field theory}

We consider firstly the effect of the Klein transformation (2.7) on strongly local observables. If one takes an arbitrary product of modular fields then there is no guarantee that after the transformations (2.5) and (2.7) are applied, the resulting products of Fermi fields will not involve the non-local Klein operator. Certainly if modular field theory is to be compared with a normal field theory then observables in the two theories should coincide. The following result is therefore reassuring:

THEOREM 4.1. After Klein transformation, strongly local observables involving modular fields consist of only normal Fermi fields and may be considered as strongly local observables in the Fermi field theory.

Proof. As was seen in the proof of Theorem 3.1 strongly local observables may be written as

$$
F(V)=\int h_{V} F_{00}^{(0)} d x_{1} \cdots d y_{1} \cdots
$$

where $F_{00}^{(0)}$ involves field polynomials of the form

$$
\phi^{\left(r_{1}\right)}\left(x_{1}\right) \cdots \phi^{\left(r_{l}\right)}\left(x_{l}\right) \phi^{*\left(t_{1}\right)}\left(y_{1}\right) \cdots \phi^{*\left(t_{n}\right)}\left(y_{n}\right),
$$

with $\sum r_{i} \equiv \sum t_{i} \bmod m ; l-n \equiv 0 \bmod m ; l+n$ even.

Upon use of the transformation (2.7), such polynomials become

$$
u^{1-r_{1}} \Phi^{r_{1}}\left(x_{1}\right) \cdots u^{1-r_{l}} \Phi^{r_{l}}\left(x_{l}\right) \Phi^{* t_{1}}\left(y_{1}\right) u^{t_{1}-1} \cdots \Phi^{* t_{n}}\left(y_{n}\right) u^{t_{n}-1} .
$$

Equation (2.6c) then allows us to write this as

$$
k u^{\left(l-n-\sum r_{i}+\sum t_{i}\right)} \Phi^{r_{1}}\left(x_{1}\right) \cdots \Phi^{r_{l}}\left(x_{l}\right) \Phi^{* t_{1}}\left(y_{1}\right) \cdots \Phi^{* t_{n}}\left(y_{n}\right),
$$

where $k$ is some phase factor involving the $m$ th primitive root of unity. Now because of the restrictions following (4.2) and the fact that $u^{m}=1$, we have demonstrated the first part of the theorem. The second part is trivial since any 
observable consisting of an even product of Fermi fields is easily shown to be strongly local. The third restriction following (4.2) requires this for strongly local observables.

We turn now to the question of identifying a suitable normal gauge theory with which modular field theory may be compared. In the normal theory, the global gauge group is implemented as follows:

$$
\Phi^{s}(x) \rightarrow \sum_{t=0}^{m-1} g^{s t} \Phi^{t}(x)
$$

where the matrices $g$ belong to an $m$-dimensional representation of the gauge group (we shall assume that it is the fundamental representation here). If we assume the vacuum is left invariant by the gauge group, then (4.5) can be shown to induce a continuous unitary representation of the gauge group on the Fockspace.

Since the classification problem for strongly local observables is as yet incomplete, we obviously cannot, as has been done in parafield theory, identify a gauge group which will select out the strongly local observables.

There may, moreover, be fundamental problems in this regard since Theorem 3.4 appears $^{5}$ to rule out the possibility of observables of second degree when $m>2$. Since the fundamental invariants of the simple Lie-groups are quadratic the existence of a "selecting" gauge group appears problematical. One possible solution to this difficulty lies in the area of non-linear representations [1]. Thus one implements the gauge group not through (4.5) but through a non-linear generalisation of it. One might hope that the linear part of the representation (namely the stability group of the related coset space) would be a group which selected out certain invariant polynomials in the usual way and that the non-linear part of the representation would leave only higher order linear invariants, invariant.

Naturally the above discussion is purely speculative and awaits further investigation for confirmation.

In view of the above difficulties we confine ourselves here to comparing modular field theory with a normal field theory having a $U(m)$ gauge symmetry.

We begin by constructing strongly local observables which, when expressed in terms of normal Fermi fields, are invariant under $U(m)$. In this regard we have

\footnotetext{
${ }^{5}$ There remains the peculiar possibility that the observables might be of higher than second order in the modular fields but of second order only in the Fermi fields.
} 
the following results:

THEOREM 4.2. Observables constructed from the following modular field polynomials are strongly local and when Klein transformed, are invariant under the gauge group $U(m)$ :

(i) $\bar{C}_{m} \bar{C}_{m}^{* \prime}$, where

$$
\begin{aligned}
\bar{C}_{m} & =\sum_{\operatorname{perm}\left(x_{0}, \ldots, x_{m-1}\right)} \psi\left(x_{0}\right) \cdots \psi\left(x_{m-1}\right) \\
& =\sum_{\operatorname{perm}(0, \ldots, m-1)} C_{m}\left(x_{0}, \ldots, x_{m-1}\right) .
\end{aligned}
$$

(ii) For $m=3$ the special case

$$
\bar{M}\left(x_{1}, x_{2}, y_{1}, y_{2}\right)=M\left(x_{1}, x_{2}, y_{1}, y_{2}\right)+M\left(x_{2}, x_{1}, y_{1}, y_{2}\right) .
$$

Proof. Consider firstly the case (i): When the ansatz (2.5) is used, $\bar{C}_{m}$ becomes (apart from a numerical factor)

$$
\bar{C}_{m}=\sum_{\text {perm }} \sum_{r_{i}=0}^{m-1} \phi^{\left(r_{0}\right)}\left(x_{0}\right) \cdots \phi^{\left(r_{m-1}\right)}\left(x_{m-1}\right) .
$$

Consider now the terms $\phi^{\left(r_{0}\right)}\left(x_{0}\right), \ldots, \phi^{\left(r_{j}\right)}\left(x_{j}\right) \cdots \phi^{\left(r_{k}\right)}\left(x_{k}\right) \cdots \phi^{\left(r_{m-1}\right)}\left(x_{m-1}\right)$ from this sum. By use of the commutation relations $(2.6 \mathrm{a})$ the $\phi^{\left(r_{j}\right)}\left(x_{j}\right)$ and $\phi^{\left(r_{k}\right)}\left(x_{k}\right)$ fields may be interchanged with the result

$$
-\eta^{r_{j}-r_{k}} \phi^{\left(r_{0}\right)}\left(x_{0}\right) \cdots \phi^{\left(r_{k}\right)}\left(x_{k}\right) \cdots \phi^{\left(r_{j}\right)}\left(x_{j}\right) \cdots \phi^{\left(r_{m-1}\right)}\left(x_{m-1}\right) .
$$

Thus if $r_{j}=r_{k}$ then this term will not be present in the sum (4.9) since this sum extends over all permutations of the spatial indices. Hence it becomes

$$
\bar{C}_{m}=\sum_{\text {perm }} \sum_{r_{i} \neq r_{j}} \phi^{\left(r_{0}\right)}\left(x_{0}\right) \cdots \phi^{\left(r_{m-1}\right)}\left(x_{m-1}\right) .
$$

If we now apply the Klein transformation (2.7), we obtain

$$
\begin{aligned}
\bar{C}_{m} & =\sum_{\text {perm }} \sum_{r_{i} \neq r_{j}} u^{1-r_{0}} \Phi^{r_{0}}\left(x_{0}\right) \cdots u^{1-r_{m-1}} \Phi^{r_{m-1}}\left(x_{m-1}\right) \\
& =\sum_{\text {perm }} \sum_{r_{i} \neq r_{j}} f\left(r_{0}, \ldots, r_{m-1}\right) \Phi^{r_{0}}\left(x_{0}\right) \cdots \Phi^{r_{m-1}}\left(x_{m-1}\right) u^{-(m(m-1)) / 2}
\end{aligned}
$$

using (2.6c), the fact that $r_{0}, \ldots, r_{m-1}$ must be a permutation of $0, \ldots, m-1$ and where

$$
f\left(r_{0}, \ldots, r_{m-1}\right) \equiv \eta^{\sum_{i>j} r_{i} r_{j}-\sum_{i=0}^{m-1}(i+1) r_{i}} .
$$

Now (4.11) may be re-expressed as

$$
\begin{gathered}
\sum_{\operatorname{perm}\left(x_{0}, \ldots, x_{m-1}\right)} \sum_{\gamma} f(\gamma(0), \gamma(1), \ldots, \gamma(m-1)) \Phi^{\gamma(0)}\left(x_{\gamma(0)}\right) \\
\ldots \Phi^{\gamma(m-1)}\left(x_{\gamma(m-1)}\right) u^{-(m(m-1)) / 2}
\end{gathered}
$$


where $\gamma$ is an arbitrary permutation of $0, \ldots, m-1$. When the anticommuting nature of the Fermi fields is used, this becomes

$$
\begin{aligned}
& \left\{\sum_{\text {perm }\left(x_{0}, \ldots, x_{m-1}\right)} \Phi^{0}\left(x_{0}\right) \cdots \Phi^{m-1}\left(x_{m-1}\right)\right\} \sum_{\gamma} \operatorname{sign}(\gamma) f(\gamma(0), \ldots, \gamma(m-1)) \\
& \times u^{-(m(m-1)) / 2} \text {. }
\end{aligned}
$$

The sum to the right of the curly brackets has been considered in an earlier publication [9] and is equal to $\eta^{c} \operatorname{det}(S)$, where $c$ is an integer and $S$ is the invertible Sylvester matrix [7] which is $m \times m$ and has the elements

$$
S_{i j}=\eta^{-i j}
$$

We may now rewrite (4.14) as

$$
\left\{\sum_{r_{0}, \ldots, r_{m-1}} \varepsilon_{r_{0}, \ldots, r_{m-1}} \Phi^{r_{0}}\left(x_{0}\right) \cdots \Phi^{r_{m-1}}\left(x_{m-1}\right)\right\} \eta^{c} \operatorname{det}(S) u^{-(m(m-1)) / 2}
$$

where $\operatorname{det}(S) \neq 0$ and $\varepsilon$ is the completely antisymmetric tensor of $m$ 'th order.

Let the quantity in brackets in (4.16) be called $D_{m}$. This has been shown [2] to transform as follows under $U(m)$ :

$$
D_{m} \rightarrow \frac{1}{\operatorname{det}(g)} D_{m}
$$

where $g$ is the matrix implementing $U(m)$ through (4.5). If we take the hermitean conjugate of (4.16), we may deduce that

$$
\bar{C}_{m} \bar{C}_{m}^{\prime *}=k D_{m} D_{m}^{\prime *}
$$

where $k$ is a real constant. Now since $G$ is unitary it follows that $\operatorname{det}(G)$ is a phase factor and hence $\bar{C}_{m} \bar{C}_{m}^{\prime *}$ is left invariant by $G$. Finally Theorem 3.2 and (4.7) show that $\bar{C}_{m} \bar{C}_{m}^{\prime *}$ can be written as a sum of field polynomials which give rise to strongly local observables and hence any observable constructed from it will obviously also be strongly local. This completes the demonstration of the first case.

In the second case we have

$$
\begin{gathered}
\bar{M}=\sum_{\substack{\operatorname{perm}\left(x_{1}, x_{2}\right) \\
\operatorname{perm}\left(y_{1}, y_{2}\right)}} \psi\left(x_{1}\right) \psi\left(x_{2}\right) \psi^{*}\left(y_{2}\right) \psi^{*}\left(y_{1}\right)-\psi\left(x_{2}\right) \psi^{*}\left(y_{2}\right) \psi^{*}\left(y_{1}\right) \psi\left(x_{1}\right) \\
+\psi^{*}\left(y_{2}\right) \psi^{*}\left(y_{1}\right) \psi\left(x_{1}\right) \psi\left(x_{2}\right)
\end{gathered}
$$


(2.3) allows us to rewrite this as

$$
\begin{gathered}
\bar{M}=\sum_{\text {perm }}\left[\sum_{r=0}^{2} \psi^{(r)}\left(x_{1}\right) \psi^{(r)}\left(x_{2}\right) \psi^{*(r)}\left(y_{2}\right) \psi^{*(r)}\left(y_{1}\right)\right. \\
-\psi\left(x_{2}\right) \psi^{*}\left(y_{2}\right) \delta\left(x_{1}-y_{1}\right)+\psi^{*}\left(y_{2}\right) \psi\left(x_{2}\right) \delta\left(x_{1}-y_{1}\right) \\
\left.-\psi^{(2)}\left(x_{1}\right) \psi^{*(2)}\left(y_{1}\right) \delta\left(x_{2}-y_{2}\right)\right] \\
=\sum_{\text {perm }}\left[\sum _ { r = 0 } ^ { 2 } \left[\psi^{(r)}\left(x_{1}\right) \psi^{(r)}\left(x_{2}\right) \psi^{*(r)}\left(y_{2}\right) \psi^{*(r)}\left(y_{1}\right)\right.\right. \\
\left.\quad-\psi^{(r)}\left(x_{2}\right) \psi^{*(r)}\left(y_{2}\right) \delta\left(x_{1}-y_{1}\right)+\delta\left(x_{2}-y_{2}\right) \delta\left(x_{1}-y_{1}\right)\right] .
\end{gathered}
$$

We show that the various order terms in this sum are invariant under $U(3)$. The scalar terms ( $\delta$ functions) are obviously so, For the second order terms we have, using (2.5), (2.6c) and the Klein transformation (2.7)

$$
\sum_{r=0}^{2} \psi^{(r)}(x) \psi^{*(r)}(y)=\sum_{s=0}^{2} \Phi^{s}(x) \Phi^{* s}(y)
$$

and, as is well known, this transforms as the fundamental invariant of $U(3)$. Consider now the fourth order terms:

$$
\begin{aligned}
& \sum_{\substack{\text { perm }\left(x_{1}, x_{2}\right) \\
\text { perm }\left(y_{1}, y_{2}\right)}} \sum_{r=0}^{2} \psi^{(r)}\left(x_{1}\right) \psi^{(r)}\left(x_{2}\right) \psi^{*(r)}\left(y_{2}\right) \psi^{*(r)}\left(y_{1}\right) \\
&= \frac{1}{9} \sum_{\text {perm }} \sum_{r=0}^{2} \sum_{s, t, v, w} \eta^{r(s+t-v-w)} \phi^{(s)}\left(x_{1}\right) \phi^{(t)}\left(x_{2}\right) \phi^{*(v)}\left(y_{2}\right) \phi^{*(w)}\left(y_{1}\right) \\
&= \frac{1}{3} \sum_{\text {perm }} \sum_{s+t=v+w} \phi^{(s)}\left(x_{1}\right) \phi^{(t)}\left(x_{2}\right) \phi^{*(v)}\left(y_{2}\right) \phi^{*(w)}\left(y_{1}\right)
\end{aligned}
$$

where we have used (2.5) and (2.6c) and the equivalence sign is modulo 3. Now since the sum is over permutations of the $x$ and $y$ spatial indices, it follows that we must have $s \neq t$ and $v \neq w$ within the summation. When the Klein transformation is applied we obtain

$$
\begin{gathered}
\frac{1}{3} \sum_{\text {perm }} \sum_{\substack{s+t=v+w \\
s \neq t v \neq w}} u^{1-s} \Phi^{s}\left(x_{1}\right) u^{1-t} \Phi^{t}\left(x_{2}\right) \Phi^{* v}\left(y_{2}\right) u^{v-1} \Phi^{* w}\left(y_{1}\right) u^{w-1}(4 \\
=\frac{1}{3} \sum_{\text {perm }} \sum_{\substack{s+t \neq v+w \\
s \neq t \\
s \neq w}} \eta^{s(1-t)} u^{2-s-t} \Phi^{s}\left(x_{1}\right) \Phi^{t}\left(x_{2}\right) \Phi^{* v}\left(y_{2}\right) \Phi^{* w}\left(y_{1}\right) \\
\times u^{v+w-2} \eta^{w(v-1)}
\end{gathered}
$$


The fact that the sum only extends over $s+t \equiv v+w$ and also (2.6c), means this may be rewritten as

$$
\frac{1}{3} \sum_{\text {perm }} \sum_{\substack{s+t \geq v+w \\ s \neq t v \neq w}} \eta^{s-w+w v-s t} \Phi^{s}\left(x_{1}\right) \Phi^{t}\left(x_{2}\right) \Phi^{* v}\left(y_{2}\right) \Phi^{* w}\left(y_{1}\right) .
$$

Consider the possible values for $s$ and $t$ in this sum: Clearly, apart from order, these are $0,10,2$ and 1,2 . Given the restriction $s+t \equiv v+w \bmod 3$, these values must be matched by the same values (apart from order) for $v$ and $w$. When the sum over spatial indices is taken into account, (4.25), becomes

$$
\begin{gathered}
\frac{1}{3} \sum_{\text {perm }} \sum_{a>b} F(a, b) \Phi^{a}\left(x_{1}\right) \Phi^{b}\left(x_{2}\right) \Phi^{* b}\left(y_{2}\right) \Phi^{* a}\left(y_{1}\right) . \\
F(a, b)=1-\eta^{b-a}-\eta^{a-b}+\eta^{a-b+b-a}=3
\end{gathered}
$$

and so therefore the sum is

$$
\sum_{\text {perm }} \sum_{a>b} \Phi^{a}\left(x_{1}\right) \Phi^{b}\left(x_{2}\right) \Phi^{* b}\left(y_{2}\right) \Phi^{* a}\left(y_{1}\right) .
$$

The sum over permutations means that this becomes

$$
\begin{aligned}
\sum_{\text {perm }} & \sum_{i, j} \Phi^{i}\left(x_{1}\right) \Phi^{j}\left(x_{2}\right) \Phi^{* j}\left(y_{2}\right) \Phi^{* i}\left(y_{1}\right) \\
\quad= & \sum_{\text {perm }} \sum_{i, j}\left[\Phi^{i}\left(x_{1}\right) \Phi^{* i}\left(y_{1}\right) \Phi^{j}\left(x_{2}\right) \Phi^{* j}\left(y_{2}\right)-\delta\left(x_{2}-y_{1}\right) \Phi^{i}\left(x_{1}\right) \Phi^{* i}\left(y_{2}\right)\right]
\end{aligned}
$$

which is manifestly invariant under $U(3)$. Given (4.8), $\bar{M}$ is, by Theorem 3.2, strongly local.

For future reference we express $\bar{M}$ in terms of the Fermi fields. By use of (4.20), (4.21) and (4.26), we deduce that

$$
\begin{aligned}
\bar{M}= & \sum_{\substack{\operatorname{perm}\left(x_{1}, x_{2}\right) \\
\operatorname{perm}\left(y_{1}, y_{2}\right)}} \sum_{i, j=0}^{2}\left[\Phi^{i}\left(x_{1}\right) \Phi^{* i}\left(y_{1}\right) \Phi^{j}\left(x_{2}\right) \Phi^{* j}\left(y_{2}\right)\right. \\
& \left.-2 \delta\left(x_{1}-y_{1}\right) \Phi^{i}\left(x_{2}\right) \Phi^{* i}\left(y_{2}\right)+\delta\left(x_{2}-y_{2}\right) \delta\left(x_{1}-y_{1}\right)\right] .
\end{aligned}
$$

A few comments are appropriate regarding Theorem 4.2: firstly, in the case that $m=2$ we are dealing with parafield theory and so the field polynomial $\left[\psi^{*}(y), \psi(x)\right]_{-}$is strongly local and invariant under $U(2)$. Secondly, one might expect that strongly local $U(m)$ invariant field polynomials could be constructed from the polynomial $M$ for arbitrary $m$. Whether this is so is not clear. We have, 
however, the following conjecture:

Conjecture: The polynomial

$$
\bar{M} \equiv \sum_{\operatorname{perm}\left(x_{1}, \ldots, x_{m-1}\right)} M\left(x_{1}, \ldots, x_{m-1}, y_{1}, \ldots, y_{m-1}\right)
$$

is strongly local and invariant under $U(m)$.

The proof of this conjecture appears to involve complicated algebraic computations and is not attempted here.

\section{The relativistic states}

We come now to the important consideration of a relativistic theory. In this case one would expect, as with the usual relativistic theory, that modular fields would be made up of two parts corresponding to positive and negative frequencies. Thus for example, one would write the free spinor modular field as [8]

$$
\psi(x)=\frac{1}{\sqrt{V}} \sum_{\mathbf{k}}\left\{e^{i\left(\mathbf{k} . \mathbf{x}-E x_{0}\right)} \sum_{t=1}^{2} u^{t}(\mathbf{k}) a_{\mathbf{k}}^{t}+e^{i\left(\mathbf{k} . \mathbf{x}+E x_{0}\right)} \sum_{t=3}^{4} u^{t}(\mathbf{k}) c_{-\mathbf{k}}^{t *}\right\}
$$

where the $u^{t}(\mathbf{k})$ are the usual Dirac spin components, $V$ is the volume appropriate to the spatial fields, and the operators $a_{\mathbf{k}}^{t}$ and $c_{\mathbf{k}}^{* *}$ are to be interpreted as particle annihilation and anti-particle creation operators respectively.

In order that the relations (2.1) be satisfied by our relativistic spatial field, the relations

$$
\begin{gathered}
a_{j} a_{k}^{*} a_{l}+a_{l} a_{k}^{*} a_{j}=\delta_{j k} a_{l}+\delta_{k l} a_{j}, \\
a_{k_{1}}^{*} a_{k_{2}} a_{k_{3}} \cdots a_{k_{m+1}}+a_{k_{3}} \cdots a_{k_{m+1}} a_{k_{2}} a_{k_{1}}^{*}=\delta_{k_{1} k_{2}} a_{k_{3}} \cdots a_{k_{m+1}}, \\
a_{k_{1}} a_{k_{2}} \cdots a_{k_{m}} a_{k_{m+1}}+a_{k_{m+1}} a_{k_{2}} \cdots a_{k_{m}} a_{k_{1}}=0
\end{gathered}
$$

which apply in the non-relativistic case, need to be extended to deal with anti-particle operators. This may be achieved by following the prescription that a creation operator $a_{k}^{*}$ in (5.2) may be replaced by an annihilation operator $c_{k}$ providing that Kronecker deltas involving the momentum labels of particles and anti-particles are removed. In otherwords $c_{k}$ acts algebraically like $a_{k}^{*}$ except with an extra degree of freedom corresponding to its status as an anti-particle operator. Similar comments apply for exchanging $a_{k}$ with $c_{k}^{*}$. With this prescription the first equation of (5.2) expands to include the following extra equations:

$$
\begin{aligned}
c_{j}^{*} c_{k} c_{l}^{*}+c_{l}^{*} c_{k} c_{j}^{*} & =\delta_{k l} c_{j}^{*}+\delta_{j k} c_{l}^{*}, \\
c_{j}^{*} a_{k}^{*} c_{l}^{*}+c_{l}^{*} a_{k}^{*} c_{j}^{*} & =0, \\
c_{j}^{*} a_{k}^{*} a_{l}+a_{l} a_{k}^{*} c_{j}^{*} & =\delta_{k l} c_{j}^{*}, \\
c_{j}^{*} c_{k} a_{l}+a_{l} c_{k} c_{j}^{*} & =\delta_{j k} a_{l}, \\
a_{j} c_{k} a_{l}+a_{l} c_{k} a_{j} & =0 .
\end{aligned}
$$


Similar extensions occur for the other two equations. It is interesting to note that when $m \geqslant 3$ these extended equations are non-trivial in the sense that one cannot just consider the anti-particle to be an ordinary particle with an "anti-particle" label. To see this, we observe that the second equation of (5.3) has no counterpart involving just particle operators (except, of course, when $m=2$ ). This non-trivial property distinguishes modular field theory from parafield theory where it is possible to consider anti-particles as simply ordinary particles with an anti-particle label [14].

We now construct a colour algebra ansatz which carries out the above prescription. We introduce the elements $e_{k}^{(r)}, e_{k}^{*(r)}, b_{k}^{(r)}, b_{k}^{*(r)}$. We then assume the colour algebra relations

$$
\begin{gathered}
e_{j}^{(r)} e_{k}^{(s)}+\eta^{r-s} e_{k}^{(s)} e_{j}^{(r)}=0, \\
e_{j}^{*(r)} e_{k}^{(s)}+\eta^{s-r} e_{k}^{(s)} e_{j}^{*(r)}=\delta_{j k} \delta^{r s} .
\end{gathered}
$$

for the $e$ and identical relations for the $b$. Between the $e$ and $b$ we assume the relations

$$
\begin{aligned}
b_{j}^{(r)} e_{k}^{*(s)}+\eta^{r-s} e_{k}^{*(s)} b_{j}^{(r)}=0, \\
b_{j}^{*(r)} e_{k}^{*(s)}+\eta^{s-r} e_{k}^{*(s)} b_{j}^{*(r)}=0 .
\end{aligned}
$$

Finally we specify the action of the $u$ operator as

$$
u^{-r} e_{k}^{(s)} u^{r}=\eta^{-r s} e_{k}^{(s)}, \quad u^{-r} b_{k}^{(s)} u^{r}=\eta^{r s} b_{k}^{(s)} .
$$

Relations such as (5.4)-(5.6) have been discussed in detail elsewhere [10] where the existence of Fock representations is demonstrated. We now define an ansatz for the particle operator $a_{j}$ and the anti-particle operator $c_{j}$ :

$$
a_{j}=\frac{1}{\sqrt{m}} \sum_{r=0}^{m-1} b_{j}^{(r)}, \quad c_{j}=\frac{1}{\sqrt{m}} \sum_{r=0}^{m-1} e_{j}^{(r)} .
$$

As in Section 2 we may also define

$$
c_{j}^{(r)} \equiv u^{-r} c_{j} u^{r}, \quad a_{j}^{(r)} \equiv u^{-r} a_{j} u^{r} .
$$

With these definitions and the equations (5.4)-(5.7) we can derive the equations

$$
\begin{gathered}
c_{j}^{(r)} c_{k}^{(s)}+c_{k}^{(s+1)} c_{j}^{(r-1)}=a_{j}^{*(r)} a_{k}^{*(s)}+a_{k}^{*(s+1)} a_{j}^{*(r-1)}=0, \\
c_{j}^{*(r)} c_{k}^{(s)}+c_{k}^{(s-1)} c_{j}^{*(r-1)}=a_{j}^{(r)} a_{k}^{*(s)}+a_{k}^{*(s-1)} a_{j}^{(r-1)}=\delta_{k j} \delta^{r s}, \\
c_{j}^{(r)} a_{k}^{*(s)}+a_{k}^{*(s+1)} c_{j}^{(r-1)}=c_{j}^{*(r)} a_{k}^{*(s)}+a_{k}^{*(s-1)} c_{j}^{*(r-1)}=0 .
\end{gathered}
$$


Substitution of the expressions (5.7) into (5.1) ${ }^{6}$ gives us our relativistic modular field. When the relations (5.9) are taken into account it is easily shown by the usual methods [8], that the fields so constructed satisfy (2.3) and hence (2.1). Moreover it is relatively easy to also see that equations (5.9) imply the extended relations such as (5.3).

We turn now to consideration of states involving anti-particles. We have already seen how the spatial modular ansatz fields are Klein transformed into Fermi fields (see equation (2.7)). In order that this be consistent with decompositions such as (5.1), we specify the following Klein transformations for the $e$ and $b$ ansatz operators:

$$
r_{j}^{(s)} \equiv u^{r-1} b_{j}^{(s)}, \quad t_{j}^{(s)} \equiv u^{1-r} e_{j}^{(s)} .
$$

This ensures that particle annihilation operators and anti-particle creation operators transform in the same way.

In the previous publication [9] it was stated that construction of the meson colour singlet state in the modular Fock-space is impossible. We shall see below that this assertion is incorrect and the relations (5.9) will be central in demonstrating the existence of such states.

Consider the operator

$$
M=\left[a_{k}^{*}, c_{l}^{*}\right]_{-}+\sum_{j=1}^{m-2} c_{n_{1}} \cdots c_{n_{j}} a_{k}^{*} c_{l}^{*} c_{n_{j}}^{*} \cdots c_{n_{1}}^{*}
$$

with $k, l \neq n_{i}, \forall i$. The use of equation (5.9) allows this to be rewritten as

$$
M=a_{k}^{*(0)} c_{l}^{*(0)}+a_{k}^{*(m-1)} c_{l}^{*(m-1)}+\sum_{j=1}^{m-2} a_{k}^{*(j)} c_{l}^{*(j)} c_{n_{1}} \cdots c_{n_{j}} c_{n_{j}}^{*} \cdots c_{n_{1}}^{*} .
$$

When this operator is applied to the vacuum state |\rangle we can use the relation

$$
c_{n_{1}} \cdots c_{n_{j}} c_{n_{j}}^{*} \cdots c_{n_{1}}^{*}|\rangle=|\rangle
$$

which was derived in the previous publication [9], to show that

$$
M|\rangle=\sum_{j=0}^{m-1} a_{k}^{*(j)} c_{l}^{*(j)}|\rangle
$$

Usage then of (5.11) and the ansatz (5.7) shows that

$$
M|\rangle=\sum_{j=0}^{m-1} r_{k}^{*(j)} t_{l}^{*(j)}|\rangle
$$

which is the colour singlet meson state.

\footnotetext{
${ }^{6}$ With an appropriate addition of a spin index.
} 
The above discussion may be extended and we may prove (modulo a technical difficulty) the general result

THEOREM 5.1. The modular Fock-space possesses all the physically relevant states of a $U(m)$ gauge theory.

The proof of this theorem may be found in the author's thesis [10] and is very close to Ohnuki and Kamefuchi's proof for parafield theory.

\section{Conclusions}

Central to our approach to modular observables has been the concept of strong locality. Apart from the causality implications, Ohnuki and Kamefuchi [14] have also pointed out that if a Hamiltonian $H(V)$ for a particular region $V$ satisfies only weak locality then the usual Heisenberg equation of motion for fields no longer holds. Given this undesirable property the strongly local observables of Theorem 2.2 would appear then to be prototypes for possible modular Hamiltonians. In view of this, an interacting field theory would seem to be implied for modular quantisation. The case of order three quantisation would appear to be most promising in this regard as it possesses a fourth order $U(3)$ invariant ( $\bar{M}$ of Theorem 4.2) which may give rise to a renormalisable theory.

Despite the above remarks it should be noted that Green [5] has constructed a quadratic Hamiltonian obeying Heisenberg's principle which can be shown [10], in its local form, to be both strongly local and invariant under $U(m)$. Such a Hamiltonian can no doubt serve as the Hamiltonian of a free modular field theory. The problem with such an operator is that it requires the operator $u$ for its expression and apparently [10] cannot be expressed purely in terms of the modular fields.

In summary, we can conclude that modular field theory is essentially equivalent to a normal field theory with a $U(m)$ gauge symmetry in which the observables have been further restricted by some, as yet unknown, requirement.

\section{References}

[1] S. Coleman, J. Wess and B. Zumino, Phys. Rev. 177 (1969), 2239.

C. Callen, S. Coleman, J. Wess and B. Zumino, Phys. Rev. 177 (1969), 2247.

[2] K. Drühl, R. Haag and J. E. Roberts, Comm. Math. Phys. 18 (1970), 204.

Y. Ohnuki and S. Kamefuchi, Progr. Theoret. Phys. 50 (1973), 258.

[3] R. Gilmore, Lie Groups, Lie Algebras and some of their applications, (Wiley, 1974) and references therein. 
[4] H. S. Green, Phys. Rev. 90 (1953), 270.

[5] H. S. Green, Austral. J. Phys. 28 (1975), 115.

[6] O. W. Greenberg, Phys. Rev. Lett. 13 (1964), 598.

O. W. Greenberg and A. M. L. Messiah, Phys. Rev. 138 (1965), B1155.

P. V. Landshoff and H. P. Stapp, Ann. Physics 45 (1967), 72.

Y. Ohnuki and S. Kamefuchi, Ann. Physics 51 (1969), 337.

These are but a selection of the more important papers.

[7] P. H. Hanus, Theory of Determinants (Ginn, Boston, 1903), p. $187 \mathrm{ff}$.

[8] G. Källén, Quantum Electrodynamics (Unwin, Springer, 1972) Chapter 3.

[9] R. Kleeman, J. Math. Phys. 24 (1983), 166.

[10] R. Kleeman, Ph.D. Thesis, University of Adelaide, 1985.

[11] See Appendix B and Section 4 Chapter 2 in [10].

[12] O. Klein, J. Phys. Radium 9 (1938), 1.

[13] Y. Ohnuki and S. Kamefuchi, Phys. Rev. 170 (1968), 1279.

Y. Ohnuki and S. Kamefuchi, "Quantum Field Theory and Parastatistics" (in Japanese), Soryushiron Kenkyu (Kyoto) 55 special issue (1977).

[14] Y. Ohnuki and S. Kamefuchi, Quantum Field Theory and Parastatistics (Univ. of Tokyo Press, Springer 1982), p. 88.

[15] V. Rittenberg and D. Wyler, Nuclear Phys. B139 (1978), 189.

M. Scheunert, J. Math. Phys. 20 (1979), 712.

R. Kleeman, J. Math. Phys. 26 (1985), 2405. 